\title{
Erratum: Eukaryotic ribosome display with in situ DNA recovery
}

Mingyue He \& Michael J Taussig

Nat. Methods 4, 281-288 (2007).

In the version of this Protocol initially published, the numbers representing primers in figure 3 , and referring to the primer table in Supplementary Methods, were incorrect. The error has been corrected in the PDF version of the Protocol. 